\title{
Growth of Core-Shell Silicon Quantum Dots in Borophosphosilicate Glass Matrix - Raman and Transmission Electron Microscopic Studies
} Dol:

10.1021/acs.jpcc.8b07316

\section{Document Version}

Accepted author manuscript

Link to publication record in Manchester Research Explorer

Citation for published version (APA):

Minami, A., Sugimoto, H., Crowe, I., \& Fujii, M. (2018). Growth of Core-Shell Silicon Quantum Dots in Borophosphosilicate Glass Matrix - Raman and Transmission Electron Microscopic Studies. The Journal of Physical Chemistry Part C: Nanomaterials, Interfaces and Hard Matter. https://doi.org/10.1021/acs.jpcc.8b07316

Published in:

The Journal of Physical Chemistry Part C: Nanomaterials, Interfaces and Hard Matter

\section{Citing this paper}

Please note that where the full-text provided on Manchester Research Explorer is the Author Accepted Manuscript or Proof version this may differ from the final Published version. If citing, it is advised that you check and use the publisher's definitive version.

\section{General rights}

Copyright and moral rights for the publications made accessible in the Research Explorer are retained by the authors and/or other copyright owners and it is a condition of accessing publications that users recognise and abide by the legal requirements associated with these rights.

\section{Takedown policy}

If you believe that this document breaches copyright please refer to the University of Manchester's Takedown Procedures [http://man.ac.uk/04Y6Bo] or contact uml.scholarlycommunications@manchester.ac.uk providing relevant details, so we can investigate your claim.

\section{OPEN ACCESS}


This document is confidential and is proprietary to the American Chemical Society and its authors. Do not copy or disclose without written permission. If you have received this item in error, notify the sender and delete all copies.

\section{Growth of Core-Shell Silicon Quantum Dots in Borophosphosilicate Glass Matrix - Raman and Transmission Electron Microscopic Studies}

\begin{tabular}{|r|l|}
\hline Journal: & The Journal of Physical Chemistry \\
\hline Manuscript ID & jp-2018-07316d.R1 \\
\hline Manuscript Type: & Article \\
\hline Date Submitted by the Author: & 21 -Aug-2018 \\
\hline Complete List of Authors: & $\begin{array}{l}\text { Minami, Akiko; Kobe University } \\
\text { Sugimoto, Hiroshi; Kobe University, Department of Electrical and Electronic } \\
\text { Engineering } \\
\text { Crowe, Iain; University of Manchester, Photon Science Institute } \\
\text { Fujii, Minoru; Kobe University, Dept of Electrical \& Electronic Eng }\end{array}$ \\
\hline
\end{tabular}

\section{SCHOLARONE ${ }^{\text {m }}$ \\ Manuscripts}




\title{
Growth of Core-Shell Silicon Quantum Dots in
}

\section{Borophosphosilicate Glass Matrix - Raman and}

\section{Transmission Electron Microscopic Studies}

\author{
Akiko Minami,,$^{\dagger}$ Hiroshi Sugimoto, ${ }^{*}{ }^{\dagger}$ Iain F. Crowe, ${ }^{*}$ and Minoru Fujii ${ }^{* \dagger}$ \\ ${ }^{\dagger}$ Department of Electrical and Electronic Engineering, Graduate School of Engineering, Kobe \\ University, Rokkodai, Nada, Kobe 657-8501, Japan \\ ${ }^{\ddagger}$ Photon Science Institute and School of Electrical and Electronic Engineering, The University of \\ Manchester, Oxford Road, Manchester, M13 9PL, UK
}

\begin{abstract}
Annealing silicon (Si)-rich borophosphosilicate glass (BPSG) at a high temperature results in the growth of core-shell Si quantum dots (QDs) composed of a boron (B) and phosphorus (P) codoped crystalline Si core and an amorphous shell made from B, Si and P (B and P codoped Si QDs) in a BPSG matrix. The amorphous $\mathrm{B}_{x} \mathrm{Si}_{y} \mathrm{P}_{z}$ shell is responsible for many superior properties of codoped Si QDs such as hydrophilicity, high resistance to hydrofluoric acid (HF) etching, stable luminescence in different environment, robustness of the luminescence for chemical
\end{abstract}


treatments, etc. In this work, we study the growth process of the amorphous shell by Raman spectroscopy and transmission electron microscopy. We show that amorphous Si particles are first grown in a BPSG matrix within $30 \mathrm{~s}$ of annealing of Si-rich BPSG. After $50 \mathrm{~s}$ annealing, a crystalline Si core appears within an amorphous Si particle. The formation of a crystalline Si core is accompanied by the formation of an amorphous $\mathrm{B}_{x} \mathrm{Si}_{y} \mathrm{P}_{z}$ shell. From the annealing time dependence of the volumes of the core and the shell, we show that supersaturated B and P are expelled to the surface of a crystalline Si core during the growth, which increases $\mathrm{B}$ and $\mathrm{P}$ concentration in an amorphous $\mathrm{B}_{x} \mathrm{Si}_{y} \mathrm{P}_{z}$ shell.

\section{Introduction,}

A colloidal solution of silicon ( $\mathrm{Si}$ ) quantum dots (QDs), often denoted as "nanocrystal ink", has been attracting significant attention because it can be a precursor for the fabrication of a silicon film for optoelectronic devices on a flexible substrate by a printed process. ${ }^{1-4}$ It has also been considered to be a promising nanophosphor for bio-imaging and -sensing ${ }^{5-8}$ due to the high biocompatibility. In any applications of the colloidal solution, agglomeration-free dispersion of Si QDs is indispensable. In order to attain the high solution dispersibility, the surface of Si QDs is usually capped by organic molecules with long alkyl chains. ${ }^{9-12}$ The molecules prevent agglomeration of QDs by the steric barrier in nonpolar solvents. Another strategy to attain solution dispersibility is by introducing surface charges, which prevent agglomeration of Si QDs in polar solvents by electrostatic repulsion. The latter scenario is important especially for biomedical applications, where the high dispersibility in aqueous media is crucial. ${ }^{7,13-15}$ 
Hydrophilic Si QDs dispersible in polar solvents can be produced by surface oxidation. ${ }^{16,17}$ However, a colloidal solution of oxidized Si QDs usually exhibits only a defectrelated blue emission, ${ }^{18-20}$ which is not suitable for biomedical applications. ${ }^{21}$ Furthermore, films produced from oxidized $\mathrm{Si}$ QDs are highly insulating and as such are not useful for optoelectronic devices. ${ }^{22}$ An interesting approach to realize hydrophilic Si QDs without oxidation is doping boron (B) on the surface. Wheeler et al. ${ }^{23}$ recently reported that trivalent acidic B sites on the QD surface have donor-acceptor bonds with polar molecules, making the QDs dispersible in polar solvents, including water. This effect is pronounced when the concentration of surface B atoms is very high. ${ }^{23,24}$ However, due to the small solid solubility of B in $\mathrm{Si}$ crystal and the preferential segregation to the $\mathrm{Si} / \mathrm{SiO}_{2}$ interface, ${ }^{25}$ preparation of $\mathrm{Si}$ QDs with very high B concentration is not straightforward.

In our previous work, ${ }^{26,27}$ we have developed a process involving thermal annealing of Si-rich borophosphosilicate glass (BPSG) to grow Si QDs with very high B concentration. During the growth of Si QDs by the phase separation of Si-rich BPSG, B and phosphorus (P) are heavily incorporated in Si QDs. A peculiarity of the process is that Si-rich BPSG, instead of Sirich borosilicate glass (BSG), is employed to incorporate $\mathrm{B}$ in the Si QDs. It has been demonstrated by atom probe tomography (APT) analyses, ${ }^{28}$ and also by ab-initio calculations, ${ }^{29}$ that simultaneous doping of P significantly increases the amount of B incorporated in Si QDs due to the charge compensation. The B and P codoped Si QDs extracted from BPSG matrices can be completely dispersed in polar solvents, such as alcohol and water, ${ }^{30}$ with no evidence of agglomeration, even years after preparation. ${ }^{26}$ The zeta potential of codoped Si QDs is $\sim-50 \mathrm{mV}$ at $\mathrm{pH} 7,{ }^{30}$ indicating that the nature of dispersion of these codoped Si QDs in polar solvents is electrostatic repulsion. It is important to note that the surface of codoped Si QDs prepared in this 
way is hydrogen $(\mathrm{H})$ terminated, because the QDs are extracted from BPSG by hydrofluoric acid (HF) etching. Therefore, the codoped Si QDs have two seemingly contradicting properties, that is, H-termination and solubility in polar solvents, owing to the very high B concentration at the surface. Thanks to the almost oxygen $(\mathrm{O})$-free surface, a film of codoped Si QDs produced by printing the ink has a semiconductor property without any post-printing processes. ${ }^{3}$

Recently, the structure of B and P codoped Si QDs was scrutinized by transmission electron microscopy (TEM) using atomically thin graphene oxide as a support film. ${ }^{31}$ The significant reduction of the background noise on a graphene oxide film, compared with a conventional amorphous carbon support film makes detailed analyses of the surface structure possible. It was found that the heavily B and P doped surface layer predicted by APT is not a B and $\mathrm{P}$ codoped crystalline Si layer, but rather an amorphous layer, the thickness of which depends on the B and P concentration of initial Si-rich BPSG. ${ }^{31}$ Therefore, the codoped Si QDs have a core-shell structure consisting of a crystalline Si core and an amorphous shell made of B, $\mathrm{Si}$ and $\mathrm{P}$ (amorphous $\mathrm{B}_{x} \mathrm{Si}_{y} \mathrm{P}_{z}$ shell). The amorphous $\mathrm{B}_{x} \mathrm{Si}_{y} \mathrm{P}_{z}$ shell is responsible for many superior properties of codoped Si QDs such as hydrophilicity, ${ }^{30}$ high resistance to HF etching, ${ }^{27}$ stable luminescence in different environment, e.g., in water ${ }^{30}$ and in air, ${ }^{32}$ robustness of the luminescence for chemical treatments, ${ }^{33}$ etc.

The purpose of this work is to study the mechanism of the formation of the amorphous $\mathrm{B}_{x} \mathrm{Si}_{y} \mathrm{P}_{z}$ shell during the growth of codoped Si QDs in BPSG by annealing Si-rich BPSG. To achieve this, we anneal Si-rich BPSG for a very short period and study the evolution of the Raman spectra and the TEM images as a function of isothermal annealing time. We show that amorphous Si particles are first grown in a BPSG matrix within $30 \mathrm{~s}$ annealing at $1200^{\circ} \mathrm{C}$. After $50 \mathrm{~s}$ annealing, a crystalline Si core appears in an amorphous particle and the core continues to 
grow with continued annealing time. During the growth of the crystalline Si core, supersaturated $\mathrm{B}$ and $\mathrm{P}$ are expelled to the surface and this forms an amorphous $\mathrm{B}_{x} \mathrm{Si}_{y} \mathrm{P}_{z}$ shell.

\section{Experimental details}

B and P codoped Si QDs were produced by a cosputtering method. Details of the preparation procedure are described in our previous papers. ${ }^{26,32,34}$ Briefly, a thick Si-rich BPSG film is deposited on a stainless steel plate or on a fused silica substrate by simultaneously sputtering $\mathrm{Si}$, $\mathrm{SiO}_{2}, \mathrm{~B}_{2} \mathrm{O}_{3}$ and phosphosilicate glass (PSG) $\left(\mathrm{SiO}_{2}: \mathrm{P}_{2} \mathrm{O}_{5}=95: 5\right.$ wt.\%). The $\mathrm{B}$ and $\mathrm{P}$ concentration in Si-rich BPSG is 0.86 and 0.62 at.\%, respectively. ${ }^{31}$ After the deposition, the Si-rich BPSG films were removed from the stainless steel plates and are collected as flakes. The flakes and Sirich BPSG films deposited on fused silica substrates were then annealed in a $\mathrm{N}_{2}$ atmosphere using a conventional tubular electric furnace. In this work, the annealing temperature was fixed to $1200^{\circ} \mathrm{C}$, while the annealing time was changed from $5 \mathrm{~s}$ to $1800 \mathrm{~s}$. After the annealing, the flakes were etched by a HF (48 wt.\%) solution and the particles grown in BPSG were liberated. Finally, the liberated particles were dispersed in methanol. For the preparation of intrinsic Si QDs, $\mathrm{Si}$ and $\mathrm{SiO}_{2}$ were simultaneously sputtered, and the deposited films (Si-rich silica) were subject to the same processes.

Raman spectra were measured using a confocal microscope $(50 \mathrm{x}$ objective lens, $\mathrm{NA}=0.8)$ equipped with a single monochromator and a charge coupled device (CCD). The excitation source was a $514.5 \mathrm{~nm}$ or $488.0 \mathrm{~nm}$ line of an Ar ion laser with excitation power of 1.0 or 0.8 $\mathrm{mW}$, respectively. For the Raman measurements of particles extracted from a BPSG matrix, a methanol solution of the particles was dropped on a gold (Au)-coated Si wafer. 
Photoluminescence (PL) spectra of samples prepared on fused silica substrates were measured by using a single grating monochromator equipped with a liquid- $\mathrm{N}_{2}$-cooled $\mathrm{InGaAs}$ diode array (OMA-V-SE, Roper Scientific). The excitation wavelength was $405 \mathrm{~nm}$. PL spectra of particles extracted from BPSG matrices and dispersed in methanol were measured by a Fluorolog-3 spectrofluorometer (HORIBA Jobin Yvon). The excitation source was a monochromatized Xe lamp (405 or $325 \mathrm{~nm}$ ) and the PL was detected by a photomultiplier (500-850 nm) and an InGaAs photodiode $(800-1500 \mathrm{~nm})$. All the optical measurements were performed at room temperature. TEM (JEM-2100F, JEOL) observations of extracted particles were performed by dropping the diluted methanol solution on a copper $(\mathrm{Cu})$ TEM mesh covered by an atomically thin graphene oxide support film. ${ }^{31}$

\section{Results and Discussion}

Figure 1a and b shows Raman spectra of Si-rich silica and Si-rich BPSG, respectively, annealed for 0-1800 s. For the Raman measurements, flakes of Si-rich silica or Si-rich BPSG obtained from a single batch were used. Therefore, the thickness and the compositions are the same for all the samples in each figure. First, as a reference, we study the evolution of Raman spectra of Sirich silica (Figure 1a). Before annealing (0 s), that is, in as-deposited Si-rich silica, the Raman spectrum exhibits very broad structures around 460 and $580 \mathrm{~cm}^{-1}$. Similar spectra are observed for 5, 10 and $20 \mathrm{~s}$ annealing, although the spectral shape is slightly modified. The spectra are apparently different from those of amorphous $\mathrm{Si}^{35-37}$ and are considered to reflect the density of state spectra of $\mathrm{SiO}_{\mathrm{x}}$. After $30 \mathrm{~s}$ annealing, the broad structure around $580 \mathrm{~cm}^{-1}$ disappears and that at $480 \mathrm{~cm}^{-1}$ is pronounced. Since the spectral shape resembles that of amorphous Si, Si-rich silica is considered to be separated into amorphous $\mathrm{Si}$ clusters and a $\mathrm{SiO}_{2}$ matrix after $30 \mathrm{~s}$ 
annealing. After $60 \mathrm{~s}$ annealing, a very small peak appears around $520 \mathrm{~cm}^{-1}$, which indicates growth of crystalline $\mathrm{Si}$ in amorphous Si clusters. The intensity of the crystalline peak increases, while that of the amorphous structure diminishes with increasing annealing time.

Figure $1 \mathrm{~b}$ shows Raman spectra of Si-rich BPSG annealed at $1200^{\circ} \mathrm{C}$ for $0-1800 \mathrm{~s}$. The overall change of the Raman spectral shape is similar to the case of Si-rich silica in Figure 1a. However, there are several important differences. First, the spectral shape for very short annealing time $(<20 \mathrm{~s})$ is different. In Si-rich BPSG, the higher wavenumber peak, around $560 \mathrm{~cm}^{-1}$ is larger than that of Si-rich silica. This may reflect the inclusion of light elements (B) in Si-rich BPSG. The spectral shape of amorphous Si clusters appearing after $20 \mathrm{~s}$ annealing is similar to that in Si-rich silica, indicating that the codoping does not strongly affect the structure of amorphous $\mathrm{Si}$ clusters. In Si-rich BPSG, a crystalline Si peak appears after $50 \mathrm{~s}$ annealing, which is earlier than the case of Si-rich silica (i.e., $60 \mathrm{~s}$ ). This is probably due to a larger diffusion coefficient of $\mathrm{Si}$ atoms in Si-rich BPSG than in Si-rich silica. The most important difference between Si-rich BPSG and Si-rich silica appears above $600 \mathrm{~cm}^{-1}$. In Si-rich BPSG, the emergence of a crystalline Si peak at $520 \mathrm{~cm}^{-1}$ is accompanied by that of a broad peak around $650 \mathrm{~cm}^{-1}$, while no such high frequency vibrational modes are observed in Si-rich silica. There are a number of known proximate local vibrational modes in this frequency range, associated with substitutional B and B-P pairs, B clusters, B-interstitial clusters, B-P clusters, etc., ${ }^{31,38-41}$ and we believe the broad, featureless peak at $650 \mathrm{~cm}^{-1}$ indicates that a combination of these is what constitutes the amorphous $\mathrm{B}_{x} \mathrm{Si}_{y} \mathrm{P}_{z}$ shell. ${ }^{31}$ We note that the intensity of the $650 \mathrm{~cm}^{-1}$ peak increases with the 520 $\mathrm{cm}^{-1}$ crystalline Si peak. 


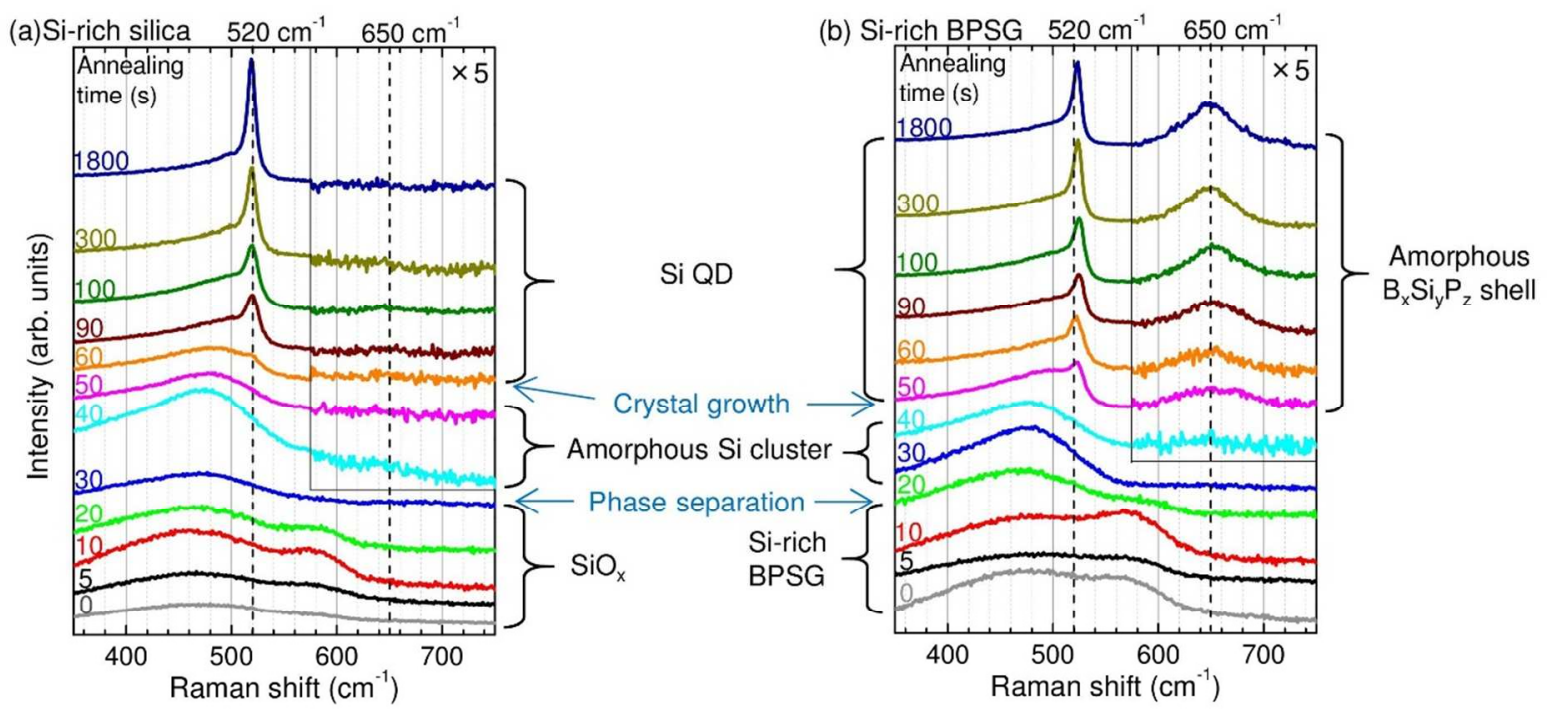

Figure 1. Raman spectra of (a) Si-rich silica and (b) Si-rich BPSG annealed at $1200^{\circ} \mathrm{C}$ for 0 $1800 \mathrm{~s}$. The annealing time is shown at the left side of the figures. For the samples annealed longer than $30 \mathrm{~s}$, the region above $575 \mathrm{~cm}^{-1}$ is multiplied by 5 to highlight the emergence and growth of the $650 \mathrm{~cm}^{-1}$ mode in the Si-rich BPSG sample.

Figure 2a and $\mathrm{b}$ shows PL spectra of Si-rich silica and Si-rich BPSG, respectively, annealed at $1200^{\circ} \mathrm{C}$ for $0-1800 \mathrm{~s}$. The spectra are normalized by the number of absorbed photons. Therefore, the intensity corresponds to the relative quantum yield. In general, the PL peak from Si-rich BPSG appears at lower energy than that of Si-rich silica. ${ }^{32}$ In order to discuss the annealing time dependence of the PL spectra more quantitatively, we plot the PL peak energy and the intensity as a function of the annealing time in Figure $2 \mathrm{c}$ and d, respectively. Below $60 \mathrm{~s}$, the PL peak energy of Si-rich silica and Si-rich BPSG is very similar $(\sim 1.27 \mathrm{eV})$, suggesting that the light emission mechanism in amorphous Si clusters is not strongly affected by B and $\mathrm{P}$ 
codoping. At $100 \mathrm{~s}$, the PL peak energy of Si-rich silica jumps up to $1.43 \mathrm{eV}$, suggesting qualitative change of the PL origin. The most plausible origin of the PL above $100 \mathrm{~s}$ is the recombination of quantum confined excitons in a crystalline Si particle. The decrease of the defect density by the crystallization may result in the steep increase of the PL intensity around 100 s. The low energy shift of the PL with further increasing the annealing temperature is explained by the growth of the crystalline $\mathrm{Si}$ particle and the weakening of the quantum confinement. After $1800 \mathrm{~s}$ annealing, the PL peak energy is $1.32 \mathrm{eV}$, which corresponds to the size of Si nanocrystal of $\sim 5 \mathrm{~nm} .{ }^{42}$

The behavior of PL from Si-rich BPSG is significantly different from that of Si-rich silica. The growth of crystalline Si particles after $50 \mathrm{~s}$ annealing results in a steep jump of the PL intensity, while the PL peak energy does not change significantly. Further increasing the annealing time results in the gradual shift to lower energy, where it finally reaches $1.08 \mathrm{eV}$ after $1800 \mathrm{~s}$ annealing. The very low energy PL of crystalline Si particles produced from Si-rich BPSG is explained by the recombination between donor and acceptor states formed by B and P co-doping. ${ }^{32}$ The PL peak reaches maximum intensity for annealing of $60 \mathrm{~s}$ and then decreases much more quickly than that of the Si-rich silica. This rapid decrease of the PL intensity with increasing size of crystalline Si particles is explained by the increased number of core-shell QDs with uncompensated carriers, in which a highly efficient, non-radiative Auger process is the most dominant recombination channel. ${ }^{43-45}$ 

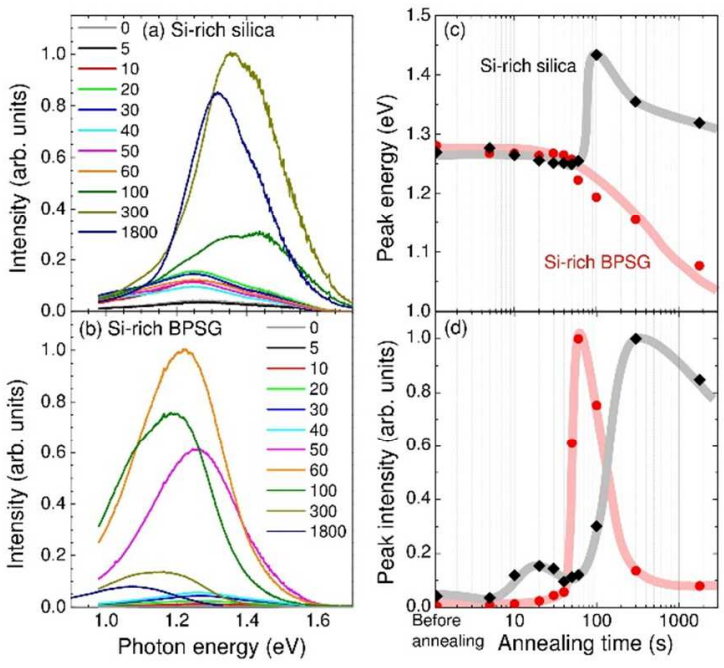

Figure 2. PL spectra of (a) Si-rich silica and (b) Si-rich BPSG annealed at $1200^{\circ} \mathrm{C}$ for $0-1800 \mathrm{~s}$. The excitation wavelength is $405 \mathrm{~nm}$. (c) PL peak energy and (d) peak intensity of Si-rich silica (black diamond) and Si-rich BPSG (red circle) as a function of annealing time.

In order to study the structure of Si particles grown by annealing Si-rich BPSG, we etched out BPSG matrices and studied the extracted particles by TEM and Raman spectroscopy. For the samples annealed below $50 \mathrm{~s}$, we could not obtain particles after the above mentioned procedure, while those annealed above $60 \mathrm{~s}$, we could retrieve particles. Figure $3 \mathrm{a}-\mathrm{d}$ shows TEM images of particles annealed for 60, 100, 300 and $1800 \mathrm{~s}$, respectively. The corresponding higher magnification images are shown in Figure 3e-h, respectively, and the corresponding Raman spectra are shown in Figure 3i-1, respectively. In Figure 3a (60 s annealed), particles around 3.5 $\mathrm{nm}$ in diameter with very small contrasts are seen. In the higher magnification image (Figure 3e), we can see that the particle has an irregular shape. The particle is predominantly amorphous but a very tiny crystalline region (about $1.6 \mathrm{~nm}$ in diameter) can be seen within the amorphous particle. The lattice fringe corresponds to $\{111\}$ planes of diamond structure indicating 
crystalline $\mathrm{Si}$. The structure, that is, an amorphous Si particle with a small crystalline Si core, is consistent with the Raman spectrum in Figure 3i. Note that particles with a clearly observable crystalline Si core were rare in the sample annealed for $60 \mathrm{~s}$. This indicates that the average size of the crystalline Si core is much smaller than that seen in Figure 3e. Comparison of the Raman spectra between Figure $3 \mathrm{i}$ and Figure $1 \mathrm{~b}$ reveals that the broad peak around $650 \mathrm{~cm}^{-1}$ almost disappears by etching. This suggests that at this growth stage, the amorphous $\mathrm{B}_{x} \mathrm{Si}_{y} \mathrm{P}_{z}$ shell is vulnerable to HF etching and is dissolved during the etching process.

Figure $3 \mathrm{~b}$ and $\mathrm{f}$ shows TEM images of particles after $100 \mathrm{~s}$ annealing. The average diameter of the particles is increased to $6.2 \mathrm{~nm}$. The crystalline core is observable in many particles and the average diameter is $3.2 \mathrm{~nm}$. The interface at the particle surface is also much clearer. The increase of the ratio of the crystalline region is consistent with the Raman spectrum in Figure $3 \mathrm{j}$, where the crystalline Si peak at $520 \mathrm{~cm}^{-1}$ grows compared to that of the sample annealed for $60 \mathrm{~s}$. In the Raman spectrum, a broad $650 \mathrm{~cm}^{-1}$ peak is clearly visible although it is slightly shifted to lower wavenumber after HF etching. The observation of the $650 \mathrm{~cm}^{-1}$ peak indicates that an amorphous $\mathrm{B}_{x} \mathrm{Si}_{y} \mathrm{P}_{z}$ shell with high resistance to HF etching is formed at this stage of annealing.

For the sample annealed for $300 \mathrm{~s}$, the particles further grow (Figure $3 \mathrm{c}$ and $\mathrm{g}$ ) with the average diameters of the particles and the crystalline cores being 8.3 and $4.7 \mathrm{~nm}$, respectively. The growth of crystalline core can also be seen in the Raman spectrum in Figure 3k. The TEM images suggest that formation of amorphous $\mathrm{B}_{x} \mathrm{Si}_{y} \mathrm{P}_{z}$ shell is completed after $300 \mathrm{~s}$ annealing, that is, core-shell Si QDs are fully formed by this stage. By increasing the annealing time to 1800 s (Figure $3 \mathrm{~d}$ and $\mathrm{h}$ ), the size of the particles and crystalline cores further increase (average particle diameter: $11 \mathrm{~nm}$, average core diameter: $7.0 \mathrm{~nm}$ ). 

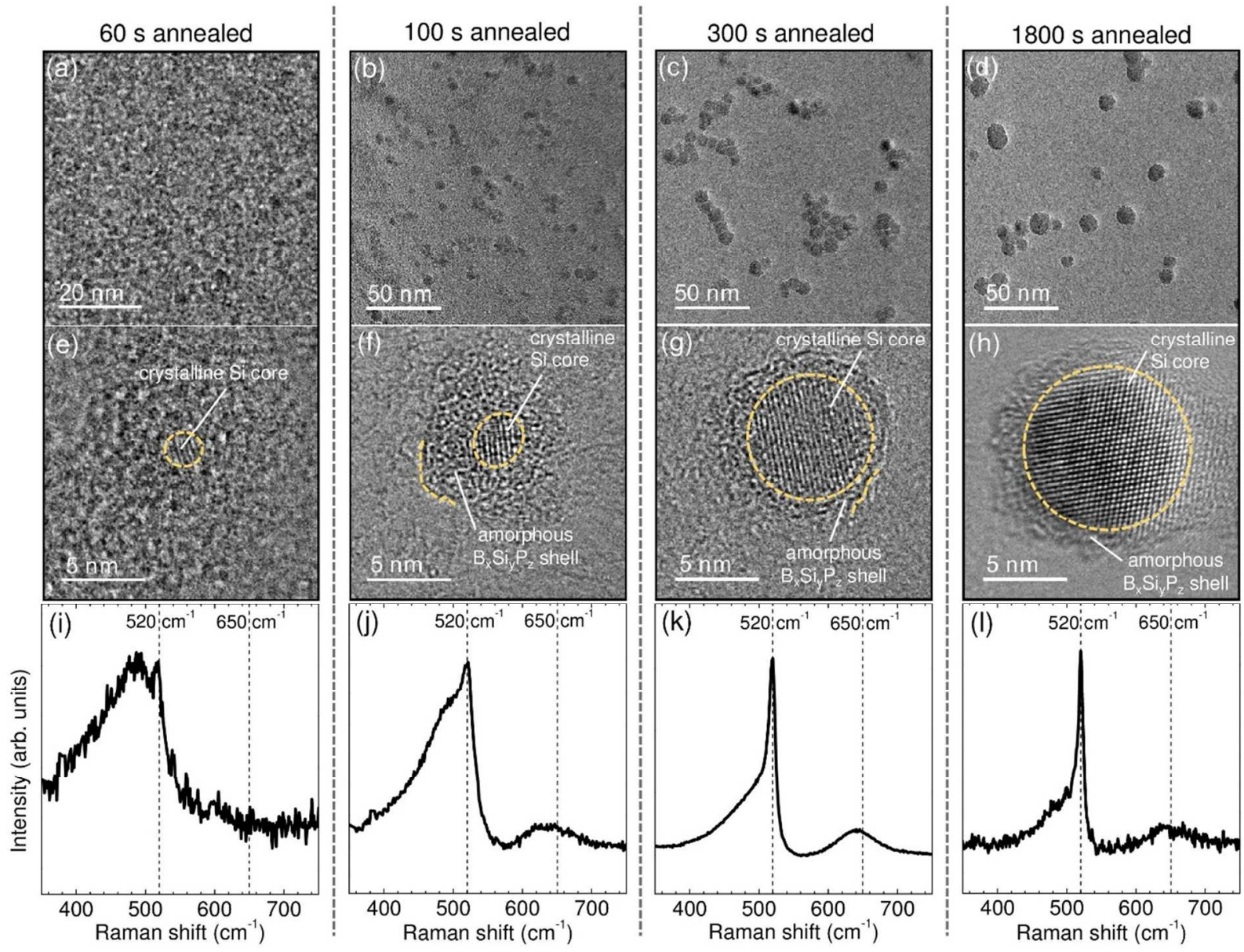

Figure 3. (a-h) TEM images and (i-l) Raman spectra of Si particles prepared by annealing Sirich BPSG for $60 \mathrm{~s}(\mathrm{a}, \mathrm{e}, \mathrm{i}), 100 \mathrm{~s}(\mathrm{~b}, \mathrm{f}, \mathrm{j}), 300 \mathrm{~s}(\mathrm{c}, \mathrm{g}, \mathrm{k})$, and $1800 \mathrm{~s}(\mathrm{~d}, \mathrm{~h}, \mathrm{l})$ at $1200^{\circ} \mathrm{C}$. The particles were extracted from BPSG matrices by HF etching and dispersed in methanol.

In Figure $4 \mathrm{a}$, the relation between the mean particle radius and the annealing time $\left(r_{p}\right.$, black circle) and that between the mean crystalline core radius and the annealing time ( $r_{c}$, red triangle) for the samples annealed longer than $100 \mathrm{~s}$ are shown. The error bars correspond to the standard deviations. Dashed lines are the results of fittings of the relations by $r_{c, p} \propto t^{x}$, where $x$ is a fitting parameter. From the fit to the particle radius, we obtain a value for $x=0.19$, which is smaller than the case of the Ostwald ripening growth process $(x=1 / 3) .{ }^{46-48}$ From the fit to the crystalline 
core radius, we obtain a value for $x(=0.27)$. The shell thickness (blue inverted triangle) estimated from the particle and core radii is also shown in Figure 4a. It increases only slightly with annealing time in the range $100 \mathrm{~s}$ to $300 \mathrm{~s}$.

Figure $4 \mathrm{~b}$ shows the annealing time dependence of the ratio of the crystalline core volume to the particle volume and that of the amorphous shell volume to the particle volume. The core volume ratio increases, while the shell volume ratio decreases with increasing the annealing time. This change of the volume ratio indicates that the composition of the shell changes during the growth of a crystalline Si core due to the following mechanism; the B and P concentration in Sirich BPSG used in this work is very high, and thus B and P are considered to reach the solubility limit within a crystalline $\mathrm{Si}$ core. On the other hand, the amorphous shell can accommodate larger concentrations of B and P due to the structural flexibility. Therefore, increasing crystalline core volume by annealing means that $\mathrm{Si}$ is preferentially consumed to form a crystalline core from the amorphous shell, and supersaturated $\mathrm{B}$ and $\mathrm{P}$ are expelled from the core to the amorphous shell. The B and P concentration in the amorphous shell thus continuously increases as the crystalline core grows. This model is supported by the experimental observation that the thickness of the amorphous $\mathrm{B}_{x} \mathrm{Si}_{y} \mathrm{P}_{z}$ shell depends on $\mathrm{B}$ and $\mathrm{P}$ concentration in Si-rich BPSG. ${ }^{31}$ 


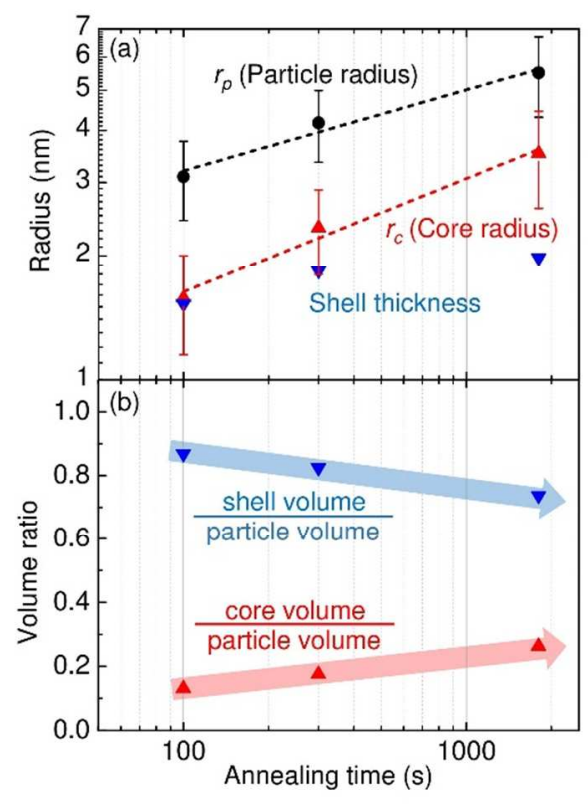

Figure 4. (a) Annealing time dependence of mean radii of particles extracted from BPSG (black circle) and crystal cores (red triangle). The radii are estimated from TEM images. The shell thickness (blue inverted triangle) estimated from the mean radii is also shown. Dashed lines are results of fitting by $r_{c, p} \propto t^{x}$, where $x$ is a fitting parameter. (b) Annealing time dependence of the ratio of core volume to particle volume (red triangle) and that of shell volume to particle volume (blue inverted triangle). The volumes are calculated from the mean radii.

Finally, we briefly discuss PL properties of codoped Si QDs extracted from BPSG matrices and dispersed in methanol (Figure 5a). Similar to the case of Si particles in BPSG, the PL intensity is the largest when the annealing time is $60 \mathrm{~s}$ and it decreases by further annealing. In Figure 5b, the PL peak energies are plotted as a function of the annealing time. We show the data obtained 1 day and 8 days after extraction. As a reference, the PL peak energy of the sample before HF etching is also shown. For the sample annealed for $60 \mathrm{~s}$, the PL peak is shifted from 
$1.22 \mathrm{eV}$ to $1.55 \mathrm{eV}$ by the etching. This significant high energy shift indicates that the quantum confinement becomes strong by the etching. A plausible explanation of the drastic change is as follows; in co-doped Si QDs in BPSG, the electron and hole wave functions are not localized in the crystalline core, but extend into the amorphous shell region, and thus the size of the whole particle determines the PL energy. However, the amorphous shell in the sample annealed for $60 \mathrm{~s}$ is vulnerable to HF etching as evidenced by the Raman spectra. The shrinkage of the shell by etching results in the stronger quantum confinement effect and the increase of the PL energy. The PL energy further increases by keeping the particles in methanol for 8 days. This is also considered to be due to the shrinkage of the amorphous shell by the oxidation in methanol.

Similar high energy shift of the PL by etching is observed for the sample annealed for $100 \mathrm{~s}$. However, since the amorphous $\mathrm{B}_{x} \mathrm{Si}_{y} \mathrm{P}_{z}$ shell is more inert to HF for the annealing condition, the change of the PL peak energy by etching is rather smaller. For the sample annealed for $1800 \mathrm{~s}$, the difference of the PL peak energy before and after etching BPSG is further reduced, indicating that the shell is almost immune to HF etching for the annealing condition.

In Figure 5b, even for the sample annealed for $1800 \mathrm{~s}$, the PL shifts slightly after keeping the particles in methanol for 8 days. This is consistent with our previous work. ${ }^{32}$ The PL property of codoped Si QDs changes during the storage in methanol for the initial a few weeks to a month after the extraction by HF etching. ${ }^{32}$ Therefore, for practical luminescence applications of codoped Si QDs, nearly a month of aging in methanol is preferable. 

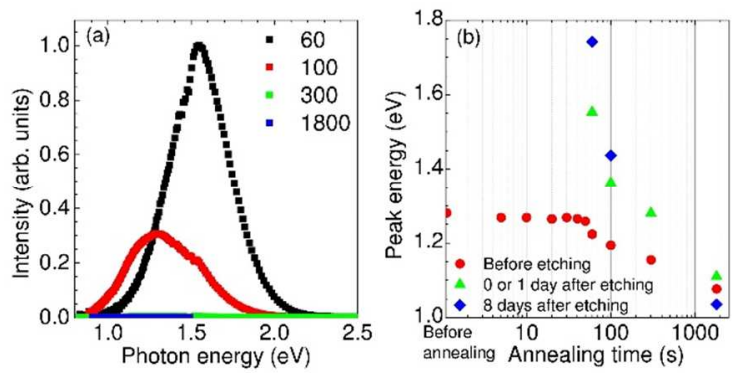

Figure 5. (a) PL spectra of Si particles extracted from Si-rich BPSG and dispersed in methanol. The annealing temperature is $1200^{\circ} \mathrm{C}$ and the annealing time is $60-1800 \mathrm{~s}$. The spectra were measured within 1 day after etching. The excitation wavelength is $405 \mathrm{~nm}$. (b) PL peak energy of codoped Si QDs in BPSG (red circle) and those extracted from BPSG and dispersed in methanol, as a function of annealing time; green triangle: within 1 day after extraction, blue diamond: 8 days after extraction.

\section{Conclusion}

The growth process of core-shell Si QDs composed of a B and P codoped crystalline Si core and an amorphous $\mathrm{B}_{x} \mathrm{Si}_{y} \mathrm{P}_{z}$ shell in a BPSG matrix obtained by annealing Si-rich BPSG was studied by Raman spectroscopy and TEM. We found that amorphous Si particles are first grown in a BPSG matrix within $30 \mathrm{~s}$ of annealing. After $50 \mathrm{~s}$ annealing, a crystalline Si core appears within an amorphous Si particle and the core continues to grow by continued isothermal annealing. Raman spectra revealed that the formation of the crystalline $\mathrm{Si}$ core is accompanied by the formation of an amorphous $\mathrm{B}_{x} \mathrm{Si}_{y} \mathrm{P}_{z}$ shell. The annealing time dependence of the volumes of the core and the shell indicated that supersaturated $\mathrm{B}$ and $\mathrm{P}$ are expelled to the surface of the 
crystalline Si core during the core growth. This increases $\mathrm{B}$ and $\mathrm{P}$ concentration in the amorphous $\mathrm{B}_{x} \mathrm{Si}_{y} \mathrm{P}_{z}$ shell.

\author{
AUTHOR INFORMATION \\ Corresponding Author \\ *E-mail: sugimoto@eedept.kobe-u.ac.jp (H.S.). \\ *E-mail: fujii@eedept.kobe-u.ac.jp (M.F.).
}

Notes

The authors declare no competing financial interest.

\begin{abstract}
ACKNOWLEDGMENT
This work was partly supported by the 2015 JST Visegrad Group (V4)-Japan Joint Research Project on Advanced Materials, JSPS KAKENHI Grant Number 16H03828 and 18K14092, and JSPS 2018 Bilateral Joint Research Projects (Japan-Australia). IFC wishes to acknowledge the support of JSPS for enabling this collaboration through a short term invitation fellowship.
\end{abstract}

\title{
REFERENCES
}


(1) Liu, C.-Y.; Holman, Z. C.; Kortshagen, U. R. Hybrid Solar Cells from P3HT and Silicon Nanocrystals. Nano Lett. 2009, 9, 449-452.

(2) Maier-Flaig, F.; Rinck, J.; Stephan, M.; Bocksrocker, T.; Bruns, M.; Kübel, C.; Powell, A. K.; Ozin, G. A.; Lemmer, U. Multicolor Silicon Light-Emitting Diodes (SiLEDs). Nano Lett. 2013, 13, 475-480.

(3) Kano, S.; Tada, Y.; Matsuda, S.; Fujii, M. Solution Processing of Hydrogen-Terminated Silicon Nanocrystal for Flexible Electronic Device. ACS Appl. Mater. Interfaces 2018, 10, 20672-20678.

(4) Ni, Z.; Ma, L.; Du, S.; Xu, Y.; Yuan, M.; Fang, H.; Wang, Z.; Xu, M.; Li, D.; Yang, J.; et al. Plasmonic Silicon Quantum Dots Enabled High-Sensitivity Ultrabroadband Photodetection of Graphene-Based Hybrid Phototransistors. ACS Nano 2017, 11, 9854 9862.

(5) Erogbogbo, F.; Yong, K.; Roy, I.; Xu, G.; Prasad, P. N.; Swihart, M. T. Biocompatible Luminescent Silicon Quantum Dots for Imaging of Cancer Cells. ACS Nano 2008, 2, 873878.

(6) Henderson, E. J.; Shuhendler, A. J.; Prasad, P.; Baumann, V.; Maier-Flaig, F.; Faulkner, D. O.; Lemmer, U.; Wu, X. Y.; Ozin, G. a. Colloidally Stable Silicon Nanocrystals with Near-Infrared Photoluminescence for Biological Fluorescence Imaging. Small 2011, 7, $2507-2516$. 
(7) Peng, F.; Su, Y.; Zhong, Y.; Fan, C.; Lee, S. T.; He, Y. Silicon Nanomaterials Platform for Bioimaging, Biosensing, and Cancer Therapy. Acc. Chem. Res. 2014, 47, 612-623.

(8) Li, Z. F.; Ruckenstein, E. Water-Soluble Poly(Acrylic Acid) Grafted Luminescent Silicon Nanoparticles and Their Use as Fluorescent Biological Staining Labels. Nano Lett. 2004, 4, 1463-1467.

(9) Mangolini, L.; Kortshagen, U. Plasma-Assisted Synthesis of Silicon Nanocrystal Inks. Adv. Mater. 2007, 19, 2513-2519.

(10) Veinot, J. G. C. Synthesis, Surface Functionalization, and Properties of Freestanding Silicon Nanocrystals. Chem. Commun. 2006, DOI: 10.1039/b607476f, 4160-4168.

(11) Hessel, C. M.; Reid, D.; Panthani, M. G.; Rasch, M. R.; Goodfellow, B. W.; Wei, J.; Fujii, H.; Akhavan, V.; Korgel, B. A. Synthesis of Ligand-Stabilized Silicon Nanocrystals with Size-Dependent Photoluminescence Spanning Visible to Near-Infrared Wavelengths. Chem. Mater. 2012, 24, 393-401.

(12) Dasog, M.; De los Reyes, G. B.; Titova, L. V; Hegmann, F. A.; Veinot, J. G. C. Size vs Surface: Tuning the Photoluminescence of Freestanding Silicon Nanocrystals Across the Visible Spectrum via Surface Groups. ACS Nano 2014, 8, 9636-9648.

(13) Ostrovska, L.; Broz, A.; Fucikova, A.; Belinova, T.; Sugimoto, H.; Kanno, T.; Fujii, M.; Valenta, J.; Kalbacova, M. H. The Impact of Doped Silicon Quantum Dots on Human Osteoblasts. RSC Adv. 2016, 6, 63403-63413. 
(14) Belinova, T.; Vrabcova, L.; Machova, I.; Fucikova, A.; Valenta, J.; Sugimoto, H.; Fujii, M.; Hubalek Kalbacova, M. Silicon Quantum Dots and Their Impact on Different Human Cells. Phys. Status Solidi 2018, DOI: 10.1002/pssb.201700597, 1700597.

(15) McVey, B. F. P.; Tilley, R. D. Solution Synthesis, Optical Properties, and Bioimaging Applications of Silicon Nanocrystals. Acc. Chem. Res. 2014, 47, 3045-3051.

(16) Kang, Z.; Liu, Y.; Tsang, C. H. A.; Ma, D. D. D.; Fan, X.; Wong, N.-B.; Lee, S.-T. WaterSoluble Silicon Quantum Dots with Wavelength-Tunable Photoluminescence. Adv. Mater. 2009, 21, 661-664.

(17) Manhat, B. A.; Brown, A. L.; Black, L. A.; Ross, J. B. A.; Fichter, K.; Vu, T.; Richman, E.; Goforth, A. M. One-Step Melt Synthesis of Water-Soluble, Photoluminescent, Surface-Oxidized Silicon Nanoparticles for Cellular Imaging Applications. Chem. Mater. 2011, 23, 2407-2418.

(18) Pi, X. D.; Liptak, R. W.; Deneen Nowak, J.; Wells, N. P.; Carter, C. B.; Campbell, S. A.; Kortshagen, U. Air-Stable Full-Visible-Spectrum Emission from Silicon Nanocrystals Synthesized by an All-Gas-Phase Plasma Approach. Nanotechnology 2008, 19, 245603.

(19) Gupta, A.; Wiggers, H. Freestanding Silicon Quantum Dots: Origin of Red and Blue Luminescence. Nanotechnology 2011, 22, 055707.

(20) Dasog, M.; Yang, Z.; Regli, S.; Atkins, T. M.; Faramus, A.; Singh, M. P.; Muthuswamy, E.; Kauzlarich, S. M.; Tilley, R. D.; Veinot, J. G. C. Chemical Insight into the Origin of Red and Blue Photoluminescence Arising from Freestanding Silicon Nanocrystals. ACS Nano 2013, 7, 2676-2685. 
(21) Hemmer, E.; Benayas, A.; Légaré, F.; Vetrone, F. Exploiting the Biological Windows: Current Perspectives on Fluorescent Bioprobes Emitting above $1000 \mathrm{~nm}$. Nanoscale Horiz. 2016, 1, 168-184.

(22) Pereira, R. N.; Niesar, S.; You, W. B.; Da Cunha, a. F.; Erhard, N.; Stegner, a. R.; Wiggers, H.; Willinger, M. G.; Stutzmann, M.; Brandt, M. S. Solution-Processed Networks of Silicon Nanocrystals: The Role of Internanocrystal Medium on Semiconducting Behavior. J. Phys. Chem. C 2011, 115, 20120-20127.

(23) Wheeler, L. M.; Kramer, N. J.; Kortshagen, U. R. Thermodynamic Driving Force in the Spontaneous Formation of Inorganic Nanoparticle Solutions. Nano Lett. 2018, 18, 18881895.

(24) Zhou, S.; Ni, Z.; Ding, Y.; Sugaya, M.; Pi, X.; Nozaki, T. Ligand-Free, Colloidal, and Plasmonic Silicon Nanocrystals Heavily Doped with Boron. ACS Photonics 2016, 3, 415422.

(25) Sze, S. M. In Physics of Semiconductor Devices, 2nd Ed.; Wiley-Interscience; New York, $1981 ; \mathrm{p} 868$.

(26) Sugimoto, H.; Fujii, M.; Imakita, K.; Hayashi, S.; Akamatsu, K. All-Inorganic NearInfrared Luminescent Colloidal Silicon Nanocrystals: High Dispersibility in Polar Liquid by Phosphorus and Boron Codoping. J. Phys. Chem. C 2012, 116, 17969-17974.

(27) Sugimoto, H.; Fujii, M.; Imakita, K.; Hayashi, S.; Akamatsu, K. Phosphorus and Boron Codoped Colloidal Silicon Nanocrystals with Inorganic Atomic Ligands. J. Phys. Chem. C 2013, 117, 6807-6813. 
(28) Nomoto, K.; Sugimoto, H.; Breen, A.; Ceguerra, A. V.; Kanno, T.; Ringer, S. P.; Wurfl, I. P.; Conibeer, G.; Fujii, M. Atom Probe Tomography Analysis of Boron and/or Phosphorus Distribution in Doped Silicon Nanocrystals. J. Phys. Chem. C 2016, 120, 17845-17852.

(29) Ossicini, S.; Degoli, E.; Iori, F.; Luppi, E.; Magri, R.; Cantele, G.; Trani, F.; Ninno, D. Simultaneously B- and P-Doped Silicon Nanoclusters: Formation Energies and Electronic Properties. Appl. Phys. Lett. 2005, 87, 173120.

(30) Sugimoto, H.; Fujii, M.; Fukuda, Y.; Imakita, K.; Akamatsu, K. All-Inorganic WaterDispersible Silicon Quantum Dots: Highly Efficient Near-Infrared Luminescence in a Wide pH Range. Nanoscale 2014, 6, 122-126.

(31) Sugimoto, H.; Yamamura, M.; Sakiyama, M.; Fujii, M. Visualizing Core-Shell Structure of Heavily Doped Silicon Quantum Dots by Electron Microscope Using Atomically Thin Support Film. Nanoscale 2018, 10, 7357-7362.

(32) Sugimoto, H.; Fujii, M.; Imakita, K.; Hayashi, S.; Akamatsu, K. Codoping n-and p-Type Impurities in Colloidal Silicon Nanocrystals-Controlling Luminescence Energy from below Bulk Band Gap to Visible Range. J. Phys. Chem. C 2013, 117, 11850-11857.

(33) Inoue, A.; Sugimoto, H.; Yaku, H.; Fujii, M. DNA Assembly of Silicon Quantum Dots/Gold Nanoparticle Nanocomposites. RSC Adv. 2016, 6, 63933-63939.

(34) Fujii, M.; Sugimoto, H.; Imakita, K. All-Inorganic Colloidal Silicon Nanocrystalssurface Modification by Boron and Phosphorus Co-Doping. Nanotechnology 2016, 27, 262001. 
(35) Voutsas, A. T.; Hatalis, M. K.; Boyce, J.; Chiang, A. Raman Spectroscopy of Amorphous and Microcrystalline Silicon Films Deposited by Low-Pressure Chemical Vapor Deposition. J. Appl. Phys. 1995, 78, 6999-7006.

(36) Iqbal, Z.; Veprek, S. Raman Scattering from Hydrogenated Microcrystalline and Amorphous Silicon. J. Phys. C Solid State Phys. 1982, 15, 377.

(37) Beeman, D.; Tsu, R.; Thorpe, M. F. Structural Information from the Raman Spectrum of Amorphous Silicon. Phys. Rev. B 1985, 32, 874-878.

(38) Newman, R. C.; Smith, R. S. Local Mode Absorption from Boron Arsenic and Boron Phosphorus Pairs in Silicon. Solid State Commun. 1967, 5, 723-726.

(39) Nazarewicz, W.; Balkanski, M.; Morhange, J. F.; Sébenne, C. Raman Scattering from Localized Vibrational Modes of Boron Impurities in Silicon. Solid State Commun. 1971, 9, 1719-1721.

(40) Adey, J.; Goss, J.; Jones, R.; Briddon, P. Identification of Boron Clusters and BoronInterstitial Clusters in Silicon. Phys. Rev. B 2003, 67, 1-5.

(41) Fujii, M.; Sugimoto, H.; Hasegawa, M.; Imakita, K. Silicon Nanocrystals with High Boron and Phosphorus Concentration Hydrophilic Shell—Raman Scattering and X-Ray Photoelectron Spectroscopic Studies. J. Appl. Phys. 2014, 115, 084301.

(42) Takeoka, S.; Fujii, M.; Hayashi, S. Size-Dependent Photoluminescence from SurfaceOxidized Si Nanocrystals in a Weak Confinement Regime. Phys. Rev. B - Condens. Matter Mater. Phys. 2000, 62, 16820-16825. 
(43) Delerue, C.; Lannoo, M.; Allan, G.; Martin, E.; Mihalcescu, I.; Vial, J. C.; Romestain, R.; Muller, F.; Bsiesy, A. Auger and Coulomb Charging Effects in Semiconductor Nanocrystallites. Phys. Rev. Lett. 1995, 75, 2228-2231.

(44) Ishii, M.; Crowe, I. F.; Halsall, M. P.; Knights, A. P.; Gwilliam, R. M.; Hamilton, B. Luminescence Quenching of Conductive Si Nanocrystals via “Linkage Emission”: Hopping-like Propagation of Infrared-Excited Auger Electrons. J. Appl. Phys. 2014, 116, 063513.

(45) Limpens, R.; Neale, N. R. Free Electron-Driven Photophysics in n-Type Doped Silicon Nanocrystals. Nanoscale 2018, 10, 12068-12077.

(46) Wagner, C. Theorie der Alterung von Niederschlägen durch Umlösen (Ostwald-Reifung). Zeitschrift für Elektrochemie, Berichte der Bunsengesellschaft für Phys. Chemie 1961, 65, $581-591$.

(47) Lifshitz, I. M.; Slyozov, V. V. The Kinetics of Precipitation from Supersaturated Solid Solutions. J. Phys. Chem. Solids 1961, 19, 35-50.

(48) Roussel, M.; Talbot, E.; Pareige, P.; Gourbilleau, F. Influence of the Supersaturation on Si Diffusion and Growth of Si Nanoparticles in Silicon-Rich Silica. J. Appl. Phys. 2013, 113, 063519 


\section{TOC Graphic}

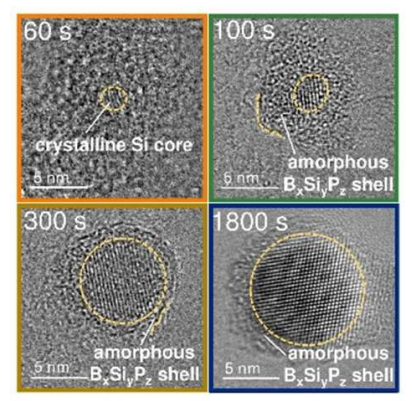

